\title{
FIELD EVALUATION OF A RAPID \\ IMMUNOCHROMATOGRAPHIC TEST 'PARACHEK-F' IN A POST-MONSOON PLASMODIUM FALCIPARUM MALARIA OUTBREAK IN VILLAGES OF SOUTH INDIA
}

\author{
Indigenous diagnostic test kit for P. falciparum
}

\author{
SUSANTA KUMAR GHOSH ${ }^{1 *}$, TIRUCHINAPALLI SUNDARAJAN SATHYANARAYAN ${ }^{1}$, \\ MALALIKOPPA VEERABHADRAPPA MURUGENDRAPPA ${ }^{2}$ AND SARALA KARUMARI SUBBARAO ${ }^{3}$ \\ Received October 11, 2001/Accepted March 9, 2002
}

\begin{abstract}
An indigenous rapid immunochromatographic test Parachek-F for diagnosis of Plasmodium falciparum malaria was evaluated by the field staff in a post-monsoon $P$. falciparum malaria outbreak in villages of district Raichur, Karnataka, South India in November 1999. The test functions based on dipstick P. falciparum histidine rich protein-2 (PfHRP-2) antigen capture assay. Of the 232 uncomplicated clinically diagnosed malaria cases, 158 $(68.1 \%)$ were positive for malaria by microscopy of JSB-stained thick blood smears. Of these, 13 were infected with $P$. vivax, 140 with $P$. falciparum and 5 had mixed infections of $P$. vivax and $P$. falciparum. Malaria patients were treated with age-specific oral doses of quinine followed by primaquine. Taking microscopy as gold standard, Parachek-F detected PfHRP-2 antigen in 136 samples (ratio 0.93) and was 93.1\% sensitive and 98.8\% specific. Positive predictive value, negative predictive value and efficacy were $99.2 \%, 89.6 \%$ and $95.2 \%$ respectively. No cross reactivity was observed with $P$. vivax infection. False negative interpretation was associated in $40 \%(10 / 25)$ lower-grade parasitaemias (parasitaemia $<100 / \mu l$ blood) where sensitivity was only $60 \%$. False positive result was associated in 1 case (1/74). Cases showing false negative results had taken presumptive treatment with chloroquine prior to the test. Careful microscopical examination on thin smears of such cases demonstrated that the morphology of the parasites was abnormal and distorted indicating the parasites were affected by chloroquine. The possible role of chloroquine resulting false negative results is suggested in this communication. Positive correlation between test bands intensity and parasite density was observed $(r=0.137 ; \mathrm{P}<0.05)$. The test is indigenously developed, rapid, simple in its application and was found suitable for field condition. Parameters like patients' conditions, history of drug intake, morphology of parasites at different developmental stages are to be considered for evaluation of such tests.
\end{abstract}

Key words: Presumptive Treatment, PfHRP-2, Immunochromatographic test, Sensitivity, Specificity

\section{INTRODUCTION}

In most parts of India malaria is unstable and has reemerged in epidemic forms with high mortality. In the last decade an increase of about $40 \%$ in Plasmodium falciparum infection has been recorded. Almost all the deaths are caused by P. falciparum infection and delayed treatment (Sharma, 1999). Malaria case detection and treatment is performed by the National Anti Malaria Programme
(NAMP) through active and passive surveillance under primary health care system. A single dose of presumptive treatment (600 $\mathrm{mg}$ chloroquine; adult dose) is administered to all fever cases at the time of taking of blood samples followed by radical treatment to the malaria positive cases. The diagnosis is based on microscopical examination of JSB-stained (Singh and Bhattacharya, 1944) whole blood by skilled personnel. In all best circumstances, it lags some important time and thus delays in the delivery of radical

\footnotetext{
${ }^{1}$ Malaria Research Centre, Field Station (ICMR), Epidemic Diseases Hospital Campus, Old Madras Road, Bangalore - 560 038, India. ${ }^{2}$ Directorate of Health and Family Welfare Services, Ananda Rao Circle, Bangalore - 560 009, India.

${ }^{3}$ Malaria Research Centre, 22-Sham Nath Marg, Delhi - 110 054, India.

"Author for correspondence: Malaria Research Centre, Epidemic Diseases Hospital Campus, Old Madras Road, Bangalore - 560038.

Email:
}

Telephone: + 91805362115 and + 91805511691 . Fax: + 91805299033 . 
treatment. The early diagnosis of $P$. falciparum malaria and institution of prompt treatment was emphasized for reducing morbidity and mortality (WHO, 1993). In such situation, test system developed by the non-microscopic antigen detection of $P$. falciparum histidine-rich protein-2 (PfHRP-2) by means of an immunological capture assay has proven to be very encouraging. The test is rapid, simple and a small amount of whole blood is required. It does not require any instrument or electricity, thus found suitable for rural setup also. The result is very specific, sensitive and can be read with the naked eyes. Evaluation of several commercially available test kits e.g., Para Sight $^{\mathrm{TM}} \mathrm{F}$ test (Karbwang et al ., 1996; Singh et al ., 1997a) and ICT Malaria ${ }^{\circledR}$ Pf test (Kumar et al., 1996; Singh et al., 1997b; Valecha et al., 1998; Ghosh et al., 2000) and Determine ${ }^{\mathrm{TM}}$ (Singh and Valecha, 2000a) have confirmed the usefulness of these rapid tests. Recently, an indigenous rapid test kit Parachek-F has been developed (Kumar et al ., 2000). This test kit, like other systems functions similar to the antigen capture assay which detects the circulating histidine-rich protein-2 (HRP-2) antigen released by $P$. falciparum (Garcia et al ., 1996). An initiative was taken by NAMP to introduce the rapid diagnostic test in the national programme. In the present study, this kit was evaluated by field staff with minimal orientation under field condition, considering microscopy as gold standard, in a post-monsoon outbreak of malaria mostly of $P$. falciparum in villages of district Raichur, Karnataka, South India.

\section{MATERIALS AND METHODS}

\section{Study area}

The Parachek-F test was conducted in two villages of two Primary Health Centres (PHCs) of district Raichur, south Karnataka during an outbreak of malaria mainly due to $P$. falciparum in November 1999. Village Ramdurg (population 2,528) of PHC Gabbur and village Masarkal (population 1,476) of PHC Masarkal were selected for the study. Generally villages in this area are large, distantly located, semi-town type, having population around 1,500 to 2,500 and well connected by good roads. Each village is surrounded by small rocky hillocks and vast irrigated land. Open dug wells and rain fed irrigation tanks are the main source of water. Escape channels from the tanks and seepage water from the hillocks form run-off stream along the village side. Malaria vectors Anopheles culicifacies, An. fluviatilis and occasionally An. stephensi were found to breed in such breeding habitats. The area is dry-deciduous with prevailing hot climate. Temperature ranges between $13{ }^{\circ} \mathrm{C}$ and $44^{\circ} \mathrm{C}$. Annual rainfall ranges from 800 to $1,200 \mathrm{~mm}$ and precipitates mainly in the post-monsoon period. Irrigation is restricted during post-monsoon period. Millets and cotton are the main cash crops. Rice is cultivated in the catchment area.

Intensive malaria control activities with fogging of malathion (5\% technical) and indoor residual spraying with deltamethrin $(2.5 \% \mathrm{wp})$ and presumptive treatment with chloroquine to all fever and fever related cases were initiated at the time of investigation. All age and sex groups including pregnant women having recent febrile illness were enrolled in the study. The test was performed also on those cases who had received presumptive treatment with chloroquine two to three days before. Such cases wanted immediate results since the reports of blood smear examination were awaited. Both $P$. vivax and $P$. falciparum species were present and both chloroquine and sulfadoxinepyrimethamine were found resistant in $P$. falciparum (Ghosh and Sathyanarayan, 2000).

\section{Work plan}

The test was conducted under field conditions by the field staff of Malaria Research Centre, Bangalore. Two scientists, one microscopist and two field health workers were involved in the test evaluation. One scientist was assigned to enroll names, history of fever, nature of ailments, any intake of anti-malarial drugs from each patient. One worker was engaged on drawing two sets of blood smears and the other performed the Parachek-F test. The microscopist stained the blood smears and examined on the spot. At the end, the second scientist correlated the thick smear results and Parachek-F test. In this way, the test procedure was blinded at each level. The final readings were rechecked by the first scientist. Local health staff and village leaders (Gram Pradhans) assisted in carrying out the tests. Verbal consent of each patient was obtained.

\section{Microscopy of JSB-stained blood smears}

Two sets of blood smears (both thick and thin) were prepared and stained with JSB stain. JSB stain is a combination of two aqueous solutions. The first solution (JSB I) contains microscopic methylene blue and the second one aqua yellowish eosin (JSB II). It is a rapid stain. First the thin smears were fixed with methanol and the thick were dehaemoglobinised by putting simply in distilled water. After completely drying both the smears were dipped into JSB II solution for 2-3 seconds and subsequently washed thoroughly in buffer water (phosphate buffer; $\mathrm{pH}$ 6.8). The slides were then dipped in JSB I solution for 45 seconds. It was again washed gently and thoroughly in the buffer. The stained slides were allowed to dry. One set was examined on the spot under a compound microscope (Zeiss KF-2, Germany) at 1,000 x magnification. The second set was 
stained in the laboratory at Bangalore and kept for reference. A smear was declared negative after counting a minimum of 200 micro fields on thick smears. Thin smears were examined for final identification of the species of parasite. All the blood smears including discordant results were thoroughly re-examined by the senior author (SKG). Parasites from each positive case were counted against 300-500 white blood cells (WBCs). The parasitaemia was calculated assuming $8,000 \mathrm{WBCs} / \mu l$ blood. $P$. falciparum cases were treated with age-specific oral doses of quinine sulphate (adult dose : $600 \mathrm{mg} 8$ hourly for 7 days) followed by a single dose of $45 \mathrm{mg}$ primaquine. $P$. vivax cases were treated with chloroquine (1,500 mg over 3 days) following by 75 $\mathrm{mg}$ primaquine in 5 days. Primaquine was not given to the infants and pregnant women.

\section{Malaria diagnosis by Parachek- $F$}

The commercial diagnostic kit Parachek-F, indigenously developed by Orchid Biomedical Systems, Verna, Goa, India was used for rapid diagnosis of $P$. falciparum malaria. The kits were supplied to the State Health Department by NAMP for field evaluation. The test system uses two mice antibodies specific against the immunodominant repeating sequence from HRP-2 molecules. One antibody is conjugated to visible colloidal gold and impregnated into the sample pad while the second one is immobilized in a line on the nitrocellulose strip. The test was performed following strict manufacturer's instruction (Fig. 1). The kits sealed in pouches were stored at $4^{\circ} \mathrm{C}$ in the $\mathrm{PHC}$ and brought to room temperature before carrying out the tests. One drop of finger pricked whole blood $(\sim 5 \mu l)$ was applied directly on the sample pad just below the arrow marks on the test strip. The strip was dipped in 4 drops of clearing buffer in a clean $12 \times 75 \mathrm{~mm}$ test tube keeping the arrows pointing downwards. In the positive samples pink coloured bands were formed along with control bands within 4-5 minutes. In the negative samples only control bands were noticed. Presence of control bands validated the tests per-

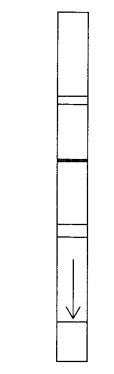

Negative test strip
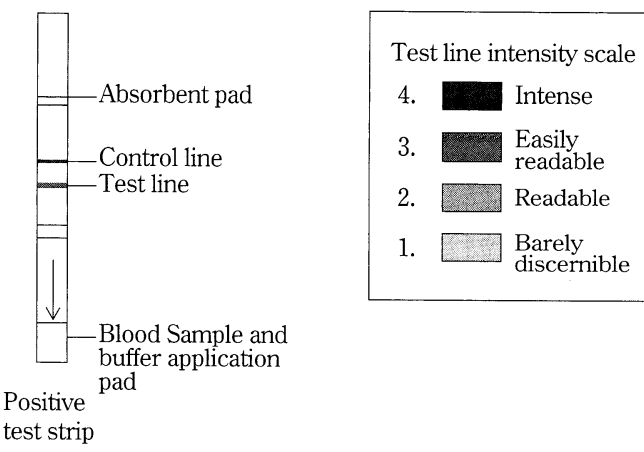

Fig. 1: Parachek-F test strip. formed. The final readings were taken after 15 minutes. The positive test bands were graded $(+1$ to +4$)$ based on the colour intensity.

\section{Data analysis}

To evaluate Parachek-F test, the result of thick smear examination was taken as gold standard. Variables like number of true positives (positive reactors in the assay with positive microscopy; TP), number of true negatives (negative reactors in the assay with negative microscopy; TN), number of false positives (positive reactors with negative microscopy; FP) and number of false negatives (negative reactors with positive microscopy; $\mathrm{FN}$ ) were measured for calculating sensitivity, specificity, positive and predictive values and efficacy of the test (Tjitra et al ., 1999). Sensitivity was thus calculated as $\mathrm{TP} /(\mathrm{TP}+\mathrm{FN})$, specificity as $\mathrm{TN} /$ $(\mathrm{TN}+\mathrm{FP})$, positive predictive value $(\mathrm{PPV})$ as $\mathrm{TP} /(\mathrm{TP}+\mathrm{FP})$ and negative predictive value (NPV) as TN/(FN $+\mathrm{TN})$. Test efficacy was defined as $(\mathrm{TP}+\mathrm{TN}) /$ total number of cases.

For the purpose of analysis, mixed infection cases having $P$. vivax and $P$. falciparum were treated as $P$. falciparum. As the subjects having $P$. falciparum gametocytes only are considered positive cases in the national programme and has epidemiological implications, these cases were considered as true positives. All P. vivax cases were treated as negative.

The correlation between test band intensity and the parasite density was analyzed using Microstat software (Ecosoft. Inc. USA) and significance was determined using F test considering $P<0.05$ to be significant.

\section{RESULTS}

Of the total 232 subjects enrolled in the study (133 from Ramdurg and 99 from Masarkal), 128 were males and 104 females. Prevalence of malaria with age wise breakdown given in Table 1 revealed that transmission of malaria was active at the study area. The youngest one was three months female and the eldest one was 80 years male. Microscopical examination on thick smears demonstrated 158 (68.1\%) were positive for malaria parasites. Of these, 13 were infected with $P$. vivax, 140 with $P$. falciparum and 5 had mixed infections of $P$. vivax and $P$. falciparum. Of the 145 P. falciparum cases, 107 were with rings only, 32 with rings and gametocytes and 6 with gametocytes only.

The Parachek-F test detected PfHRP-2 antigen in 136 (ratio 0.93) P. falciparum patients and was non-reactive to $P$. vivax cases (Table 2). Considering $P$. falciparum rings only, the test detected 99 of 107 cases (ratio 0.92); while in the group with gametocytes only 4 out of 6 cases were detected (ratio 0.66). All the 32 cases having concomitant 
Table 1 Prevalence of malaria in the affected villages of district Raichur, south Karnataka in November 1999

\begin{tabular}{|c|c|c|c|c|c|c|c|c|}
\hline $\begin{array}{l}\text { Patients age } \\
\text { Groups (years) }\end{array}$ & $\begin{array}{l}\text { Blood smear } \\
\text { examined }\end{array}$ & $\begin{array}{l}\text { Positive for } \\
\text { malaria }\end{array}$ & P. vivax & P. falciparum & $\begin{array}{l}\text { P. vivax }+ \\
\text { P. falciparum }\end{array}$ & $\begin{array}{l}\text { Slide Positivity } \\
\text { Rate }\end{array}$ & $\begin{array}{l}\text { Slide falciparum } \\
\text { Rate }\end{array}$ & $\begin{array}{l}\text { P. falciparum } \\
\text { Percentage }\end{array}$ \\
\hline$\leq 1$ & 7 & 3 & 2 & 1 & 0 & 42.85 & 14.28 & 33.33 \\
\hline$>1-\leq 4$ & 17 & 12 & 1 & 10 & 1 & 70.58 & 64.70 & 91.66 \\
\hline$>4-\leq 8$ & 24 & 21 & 1 & 19 & 1 & 87.50 & 83.33 & 95.23 \\
\hline$>8-\leq 14$ & 31 & 21 & 3 & 17 & 1 & 67.74 & 58.06 & 85.71 \\
\hline$>14$ & 147 & 98 & 5 & 91 & 2 & 66.66 & 63.26 & 94.89 \\
\hline $\begin{array}{l}\text { Pregnant } \\
\text { Women }\end{array}$ & 6 & 3 & 1 & 2 & 0 & 50.00 & 33.33 & 66.66 \\
\hline Total & 232 & 158 & 13 & 140 & 5 & 68.10 & 62.50 & 91.77 \\
\hline
\end{tabular}

Table 2 Performance of Parachek-F assay in 232 clinically diagnosed malaria patients

\begin{tabular}{lrcc}
\hline & \multicolumn{3}{c}{ No. Plasmodia positive by } \\
\cline { 2 - 4 } & Thick smear & Parachek-F & Ratio \\
\hline P. falciparum & 140 & & \\
P. vivax & 13 & 0 & \\
P. vivax +P. falciparum & 5 & 5 & \\
Total positive & 158 & 136 & 0.86 \\
Total negative & 74 & 96 & \\
\hline P. falciparum only & & & \\
Rings only & 107 & 99 & 0.92 \\
Rings + Gametocytes & 32 & 32 & 1.00 \\
Gametocytes only & 6 & 4 & 0.66 \\
\hline Total positive taking all & 145 & 136 & 0.93 \\
P. falciparum cases & & & \\
\hline
\end{tabular}

rings and gametocytes showed positive to both microscopy and PfHRP-2 antigen (ratio 1.00). Thus the sensitivity of PfHRP-2 assay was $93.1 \%$, the specificity $98.8 \%$, the positive predictive value $99.2 \%$, the negative predictive value $89.6 \%$ and the test efficacy was $95.2 \%$ respectively. Prior to the test, 19/135 TP, 10/10 FN, 1/1 FP and 12/86 TN (total 42/232) cases consumed chloroquine as presumptive treatment (Table 3).

Details of parasitaemia in the $P$. falciparum cases and related sensitivity are summarized in Table 4. Parasitaemia ranged from 17 to 57,120 parasites $/ \mu l$ blood. Compared to microscopy, Parachek-F failed to detect infection in 10 of 25 patients $(40 \%)$ with low parasitaemia $(<100$ parasites $/ \mu l$ blood) where 8 were with rings only and 2 with gametocytes only. Thus, the test demonstrated $60 \%$ sensitive in lower-grade parasitaemias and $100 \%$ in cases having $>100$ parasites $/ \mu l$ blood. Careful microscopical examination on thin smears in the lower-grade parasitaemias revealed gross distortion of the parasite morphology. The rings were very small, nugget shaped, chromatin and cytoplasm were compact due to lack of vacuole. The morphology of the gametocytes was also altered having oval and slightly elongated shapes. The pigments and chromatin were dispersed, dis-
Table 3 Efficacy of Parachek-F test

\begin{tabular}{lcr}
\hline & \multicolumn{2}{c}{ Microscopic diagnosis } \\
& + & - \\
\hline Parachek-F + & $135(\mathrm{TP})$ & $1(\mathrm{FP})$ \\
diagnosis - & $10(\mathrm{FN})$ & $86(\mathrm{TN})$ \\
\hline
\end{tabular}

Sensitivity $=135 /(135+10)=0.931 ;$ Specificity $=$ $86 /(86+1)=0.988 ;$ Positive predictive value $=$ $135 /(135+1)=0.992 ;$ Negative predictive value $=$ $86 /(86+10)=0.896 ;$ Efficacy $=(135+86) /(135+$ $10+1+86)=0.952$.

All $P$. falciparum gametocyte cases considered as positive $P$. vivax cases considered as negative. TP - True positive, FP - False positive, FN - False negative, TN - True negative

Table 4 Sensitivity of Parachek-F test by parasitaemia

\begin{tabular}{cccc}
\hline $\begin{array}{l}\text { Parasitaemia/ } \\
\mu l \text { blood }\end{array}$ & $\begin{array}{c}\text { Thick smear } \\
\text { diagnosis }\end{array}$ & $\begin{array}{c}\text { Parachek-F } \\
\text { diagnosis }\end{array}$ & Sensitivity (\%) \\
\hline $17-100$ & 25 & 15 & 60 \\
$101-500$ & 14 & 14 & 100 \\
$501-1,000$ & 16 & 16 & 100 \\
$1,001-2,000$ & 53 & 53 & 100 \\
$2,001-5,000$ & 23 & 23 & 100 \\
$>5,000$ & 12 & 12 & 100 \\
All P. falciparum & 145 & 146 & 99.2 \\
cases & & & \\
\hline
\end{tabular}

tributed in the cytoplasm.

Details of correlation between test band intensity and stage wise parasite density is shown in Table 5. Positive correlation $(r=+0.137 ; P<0.05)$ was noticed in all stages of parasite except in cases having only gametocytes $(r=-0.749$; $P>0.05)$ and band intensity grade $2(r=-0.306 ; P>0.05)$.

\section{DISCUSSION}

In the study area malaria was not a serious problem in the recent past (NAMP, 1998). In the beginning of the study, there was a confusion of the cause of outbreak even though 
Table 5 Correlation between parasite density of $P$. falciparum and colour intensity of test bands along with different stages of parasite in the test samples.

\begin{tabular}{|c|c|c|c|c|c|}
\hline $\begin{array}{l}\text { Parasite Density } \\
\text { Mean } \pm \mathrm{SD} \\
\text { (Range) }\end{array}$ & $\begin{array}{l}\text { Band } \\
\text { Intensity }\end{array}$ & Stage of Parasite & $r$ value & F ratio & $P$ value \\
\hline $\begin{array}{l}\text { Band wise } \\
56.60 \pm 25.27 \\
(17-106)\end{array}$ & 0 & $\mathrm{R}(\mathrm{n}=8), \mathrm{G}(\mathrm{n}=2)$ & +0.055 & 50.15 & 0.0001 \\
\hline $\begin{array}{l}843.53 \pm 1,821.66 \\
(0-9,420)\end{array}$ & 1 & $\begin{array}{l}\text { Neg. }(n=1), R(n=18) \\
G(n=2), R G(n=5)\end{array}$ & +0.330 & 5.56 & 0.0223 \\
\hline $\begin{array}{l}2,379.67 \pm 7,384.31 \\
(55-41,320)\end{array}$ & 2 & $\begin{array}{l}R(n=25), G(n=1) \\
R G(n=4)\end{array}$ & -0.306 & 3.11 & 0.0831 \\
\hline $\begin{array}{l}2,180.59 \pm 4,691.93 \\
(54-27,290)\end{array}$ & 3 & $\mathrm{R}(\mathrm{n}=21), \mathrm{RG}(\mathrm{n}=11)$ & +0.296 & 6.89 & 0.0109 \\
\hline $\begin{array}{l}6,686.79 \pm 2,133.01 \\
(220-57,120)\end{array}$ & 4 & $\begin{array}{l}R(n=35), G(n=1) \\
R G(n=12)\end{array}$ & +0.240 & 14.56 & 0.002 \\
\hline $\begin{array}{l}\text { Stage wise } \\
605.0 \pm 1,293.54 \\
(45-3,245)\end{array}$ & $(0-4)$ & G only $(n=6)$ & -0.749 & 1.30 & 0.279 \\
\hline $\begin{array}{l}3,776.11 \pm 9,388.23 \\
(17-57,120)\end{array}$ & $(0-4)$ & R only ( $\mathrm{n}=107)$ & +0.812 & 17.28 & 0.0004 \\
\hline $\begin{array}{l}2,461.90 \pm 4,693.75 \\
(104-27,290)\end{array}$ & $(1-4)$ & $\mathrm{RG}(\mathrm{n}=32)$ & +0.159 & 8.78 & 0.004 \\
\hline $\begin{array}{l}33,254 \pm 8,384.81 \\
(17-57,120)\end{array}$ & $(0-4)$ & $\begin{array}{l}\text { All P. falciparum } \\
\text { cases }\end{array}$ & +0.137 & 23.17 & 0.0002 \\
\hline
\end{tabular}

R - rings, G - gametocytes, Neg. - Negative for malaria.

all anti-malarial measures had initiated. The Parachek-F test clearly identified that the outbreak was due to $P$. falciparum malaria showing high sensitivity of $93.1 \%$ which is in agreement with similar commercial kits like Para Sight $^{\mathrm{TM}}-\mathrm{F}$ (Beadle et al ., 1994; Karbwang et al ., 1996; Singh et al ., 1997a) and ICT-Pf (Kumar et al ., 1996; Singh et al ., 1997 b; Valecha et al., 1998; Ghosh et al., 2000). Other values like specificity, positive and negative predictive values and efficacy of the test were comparable. Kumar et al . (2000) also demonstrated similar results with the same Parachek-F kit.

As compared to Parachek-F, Para Sight ${ }^{\mathrm{TM}}-\mathrm{F}$ and ICTPf have several washing processes. In this regard, Parachek$\mathrm{F}$ is very simple and easy to operate in the field by the health workers (Fig. 1). Due to its simplicity, it is costeffective also. Each kit costs approximately U.S.\$ 0.64 which is cheaper than other commercial kits viz. Para Sight $^{\mathrm{TM}}-\mathrm{F}$ and ICT-Pf (between U.S.\$ 2.91 and 3.33) but higher than the suggested price of U.S.\$ 0.40 by H. Engers (Singh and Valecha, 2000a). To make accessible, efforts should be made to produce such kits locally specially in the developing countries where malaria (mainly $P$. falciparum) is a real problem. In India, cold chain system has been provided in each PHC under primary health care. Thus, there will not be any problem to introduce this test system in the national programme.

There are some limitations in this test for poor interpretation in lower-grade parasitaemias. Low parasitaemia are most common in many areas where anti-malarial drugs are easily available and people take self-medication. Such situation is very common among travellers who are advised to cover with chemoprophylaxis (WHO, 1996). Funk et al . (1999) reported false negative results among travellers up to 72.0\% in ICT-Pf and 29.6\% cases in Para Sight F where parasitaemia level was $<0.1 \%$. They did not find these kits suitable for self-diagnosis. Beadle et al . (1994) observed in a field test in Kenya that sensitivity and specificity markedly decreased in lower-grade parasitaemias. We have observed false negative results in $40 \%$ cases having parasitaemia level <100 parasites/ $\mu l$ blood. Karbwang et al . (1996) observed a lack of correlation between the amount of PfHRP-2 antigen and small ring form parasitaemia. Better correlation was observed with the trophozoite load (late rings). Beadle et al . (1994) reported that PfHRP-2 antigen is produced in trophozoites and early gametocytes. Recently, PfHRP-2 has been reported to mediate haemozoin formation, which could be inhibited by chloroquine (Sullivan et al., 1996). Pandey et al. (1997) have demonstrated that 
hexapeptide repeats Ala-His-His-Ala-Ala-Asp in PfHRP-2 are heme binding sites in this protein. It could be possible that in the early developmental stages of the parasites, PfHRP-2 molecules are not expressed due to the effect of chloroquine and demonstrate false negative results. The patients who showed false negative results showed clinical symptoms for malaria and had consumed presumptive treatment with chloroquine prior to the test. This was confirmed by the health workers who administered the presumptive treatment. Further, the morphology of the parasites in these cases clearly indicated that they were affected by chloroquine as described by Macomber and Sprinz (1967). Karbwang et al. (1996) observed very low specificity of $50 \%$ in Para Sight ${ }^{\mathrm{TM}} \mathrm{F}$ test for detection of multi-drug resistant $P$. falciparum treatment failure as against $60 \%$ in our study cases underwent presumptive treatment. This is possibly due to the drug affected parasitaemia phenomenon.

One false positive case (1/74) detected by Parachek-F was treated 11 days before the test. This is possible when persistence of antigenemia in the patient persists even after the curative treatment (Singh et al ., 1997a, b; Valecha et al ., 1998; Kumar et al., 2000). Recently, Grobusch et al. (1999) has reported false-positive results in the presence of rheumatoid factors. False-positive test results could lead to inappropriate treatment of other important causes of fever. However, this should not be taken as limitation to the test because timely treatment in many sub-microscopic level parasitaemias in known malarious areas may prove fruitful.

There are mixed reports on the correlation between the test band intensity and parasite density. Some reported positive correlation (Kumar et al., 1996; Ghosh et al., 2000), while others negative correlation (Singh et al ., 1997a, Valecha et al., 1998). Kumar et al. (2000) reported negative correlation with the same kit as against positive correlation in our study.

Recently, test kits for diagnosis of both $P$. falciparum and $P$. vivax are available commercially. One such kit (ICT P.f./P.v.) was evaluated by Singh et al. (2000b) in Madhya Pradesh, Central India. The test was $97.5 \%$ sensitive and $88 \%$ specific for $P$. falciparum. For $P$. vivax the sensitivity was only $72 \%$ and $99 \%$ specific. The authors concerned about the decline in sensitivity $<300$ parasites $/ \mu l$ for $P$. falciparum. The test failed to diagnose $P$. vivax infection when the parasitaemia was $\leq 1500$ parasites $/ \mu l$. Tjitra et al. (1999) also made similar observation.

In conclusion, rapid immunodiagnostic tests are very useful in the field and in emergencies even having some limitations, because all test systems do have some selflimiting factors. Proper caution has to be taken for those subjects undertaking chemoprophylaxis (especially pregnant women and travellers). The test systems have addi- tional advantage as the results can be kept as reference for a fairly long period of time. All the test cards of our study are easily legible even after more than one and half years of testing. For evaluation of such test systems, it is very important to consider parameters like patients' condition, history like drug intake, parasite morphology, etc. However, this cannot replace the microscopical diagnosis for routine epidemiological studies where different stages of parasites, their density etc. are important components, which cannot be performed by such test systems.

\section{ACKNOWLEDGEMENTS}

The authors thank the District Malaria Officer, district Raichur, the Medical Officers, Primary Health Centres Gabbur and Masarkal and staff of Malaria Research Centre, Bangalore for assistance in the study.

\section{REFERENCES}

1) Beadle, C., Long, G.W., Weiss, W.R., McElroy, P.D., Maret, S.M., Oloo, A.J. and Hoffman, S.L. (1994): Diagnosis of malaria by detection of Plasmodium falciparum HRP-2 antigen with a rapid dipstick antigen-capture assay. Lancet, $343,564-567$

2) Funk, M., Schlagenhauf, P., Tschopp, A. and Steffen, R. (1999): Mala Quick ${ }^{\mathrm{TM}}$ versus Para Sight $\mathrm{F}^{\circledR}$ as a diagnostic aid in travellers' malaria. Trans. Roy. Soc. Trop. Med. Hyg., 93, 268-272

3) Garcia, M., Kirimoama, D., Marlborough, D., Leafaria, J. and Rieckmann, K.H. (1996): Immunochromatographic test for malaria diagnosis. Lancet, 347, 1549

4) Ghosh, S.K., Titus Burk, E., Valecha, N., Murugendrappa, M.V. and Sharma, V.P. (2000): Evaluation of a rapid immunochromatographic test for detection of Plasmodium falciparum in Karnataka, India. J. Parasit. Dis., 24, 39-42

5) Ghosh, S.K. and Sathyanarayan, T.S. (2000): In vivo response of Plasmodium falciparum to chloroquine and sulfadoxine-pyrimethamine in Karnataka. Paper presented in the 'Fourteenth National Congress of Parasitology', 2326 April, New Delhi, India. Abstract No. O-3.10, p-56

6) Grobusch, M.P., Alpermann, U., Schwenke, S. and Jelinek, T. (1999): Determination of false positive rapid malaria tests in patients with rheumatoid factor. Lancet, 353, 297

7) Karbwang, J., Tasanor, O., Kanda, T., Wattanagoon, Y., Ibrahim, M., Nabagchng, K., Thanavibul, A. and Rooney, W. (1996): Para Sight ${ }^{\mathrm{TM}}-\mathrm{F}$ test for the detection of treatment failure in multi-drug resistant Plasmodium falciparum malaria. Trans. Roy. Soc. Trop. Med. Hyg., 90, 513515

8) Kumar, A., Sharma, V.P., Thavaselvam, D. and Sumodan, P.K. (1996): Clinical trials of a new immunochromatographic test for diagnosis of Plasmodium falciparum in Goa. Indian J. Malariol., 33, 166-172 
9) Kumar, A., Sumodan, P.K. and Sharma, V.P. (2000): Clinical trials of an indigenous diagnostic kit Parachek-F for the diagnosis of Plasmodium falciparum malaria in Goa. J. Parasit. Dis., 24, 43-45

10) Macomber, P.B. and Sprinz, H. (1967): Morphological effects of chloroquine on Plasmodium berghei in mice. Nature (London), 214, 937-938

11) National-Anti Malaria Programme (1998): NAMP Annual Report for Karnataka - 1998. Regional Office for Health and Family Welfare, Government of India, Bangalore

12) Pandey, A.V., Joshi, R.M., Tekwani, B.L., Singh, R. and Chauhan, V.S. (1997): Synthetic peptides corresponding to repetitive sequence of malarial histidine rich protein bind heme and inhibit haemozoin formation in vitro. Mol. Biochem. Parasit., 90, 281-287

13) Sharma, V.P. (1999). Current scenario of malaria in India. Parasitologia, 41, 349-353

14) Singh, J. and Bhattacharya, L.M. (1944): Rapid staining of malarial parasites by a water soluble stain. Indian Med. Gaz.., 79, 102-104

15) Singh, N., Singh, M.P. and Sharma, V.P. (1997a): Dipstick antigen capture assay for malaria detection in a forested area of central India (M.P.). Am. J. Trop. Med. Hyg., 56, 188-191

16) Singh, N., Valecha, N. and Sharma, V.P. (1997b): Malaria diagnosis by field workers using an immunochromatic test. Trans. Roy. Soc. Trop. Med. Hyg., 91, 396-397

17) Singh, N. and Valecha, N. (2000a): Evaluation of a rapid diagnostic test, 'Determine ${ }^{\mathrm{TM}}$ malaria pf', in epidemicprone forest villages of central India (Madhya Pradesh). Ann. Trop. Med. Parasit., 94, 421-427
18) Singh, Neeru, Saxena Ajay and Valecha Neena (2000b): Field evaluation of the ICT malaria P.f/P.v immunochromatographic test for diagnosis of Plasmodium falciparum and $P$. vivax infection in forest villages of Chhindwara, central India. Trop. Med. Int. Hlth., 5, 765-770

19) Sullivan, D.J., Gluzman, I.Y. and Goldberg, D.E. (1996): Plasmodium haemozoin formation mediated by histidine rich proteins. Science (Washington, D.C.) 271, 219-221

20) Tjitra, E., Suprianto, S., Dyer, M., Currie, B.J. and Anstey, N.M. (1999): Field evaluation of the ICT malaria P.f/P.v. immunochromatographic test for detection of Plasmodium falciparum and Plasmodium vivax in patients with a presumptive clinical diagnosis of malaria in eastern Indonesia. J. Clin. Microbiol., 37, 2412-2417

21) Valecha, N., Sharma, V. P and Usha Devi, C. (1998): A rapid immunochromatographic test (ICT) for diagnosis of Plasmodium falciparum. Diag. Microbiol. Infec. Dis., 30, 257-260

22) W.H.O. (1993): A Global Strategy for Malaria Control. World Health Organization. Geneva, p-1

23) W.H.O. (1996): Management of uncomplicated malaria and the use of antimalarial drugs for the protection of travellers. Report of an informal consultation. Geneva 18-21 September 1995. WHO/MAL/96, 1075, 98 